\title{
A Conversation with Huda Zoghbi
}

\author{
INTERVIEWER: JAN WITKOWSKI \\ Cold Spring Harbor Laboratory
}

\begin{abstract}
Huda Zoghbi is a Professor in the Departments of Pediatrics, Molecular and Human Genetics, Neuroscience, and Neurology at Baylor College of Medicine, the Director of the Jan and Dan Duncan Neurological Research Institute at Texas Children's Hospital, and an Investigator at the Howard Hughes Medical Institute.
\end{abstract}

Jan Witkowski: The subject of your work is enticing: fine-tuning protein...

Dr. Zoghbi: Fine-tuning protein levels for neurological health. So, what does it really mean? What does it translate to in scientific terms and in practical terms? This is a principle that - in my experience, having been now in research for 30 years - applies to all of the neurological disorders that I have discovered, from childhood diseases all the way to adult, aging-related disorders: That's the overarching principle: protein levels matter for brain health. Actually, for the first 10 - or even 20 - years of my career, that was not anywhere on my radar screen, but it was really through the data and the experiments that we've done that it became obvious.

Jan Witkowski: When you're talking about protein levels, are you meaning levels of particular proteins?

Dr. Zoghbi: Correct. There are examples of proteins that the brain is exquisitely sensitive to their level. For some of these, a small change in the $10 \%-20 \%$ range will make the older brain highly vulnerable.

Jan Witkowski: This is a change of level, not a mutation in that protein?

Dr. Zoghbi: Well, it can be either. Some examples are pure change in level without any mutation - this is some of the recent data we have - and sometimes a mutation will lead to enhanced level and/or function of the protein, so you can get there in more than one way. You can get there by a mutation in the gene encoding the protein itself, or you can get there by changing the level of a regulator of that protein.

Jan Witkowski: An example that comes to my mind is in spinal muscular atrophy where you have multiple copies of SMN2 [Survival of Motor Neuron 2], and you've got more protein.

Dr. Zoghbi: In spinal muscular atrophy you actually have multiple copies of the SMN2 gene, but there's one copy on each chromosome of $S M N 1$ - the one that's properly spliced - that makes most of the protein. When the mutations in SMN1 happen, you inactivate the protein, so you're left with very little protein, and the patients have disease. But their disease is modified by the level of the protein made from paralog, SMN2. If you have very little, then it's a very severe phenotype. If you make a little bit more due to more copies of $S M N 2$, it is still severe but more moderate. If you have a lot more, you make it milder. That's due to a splicing mutation: If a mutation leads to abnormal splicing, you don't make a full-length protein. So the new therapythe antisense - allows that exon to be properly spliced, leading to more of the protein to be made, which rescues the symptoms.

I studied a really very rare disease; nobody probably heard of it, an ataxia. It's a balance disorder. It affects coordination and walking and it happens later in life, typically when the person is a mature adult. The reason we studied it is because it's inherited. It's Mendelian, so it was easy to track. My thought was that if we learn something from it, it might inform us about the broader class of neurodegenerative diseases. In 1993, Harry Orr and I identified the gene and found it is due to a repeat expansion: a trinucleotide repeat of CAGs that encode glutamine. In you or I, we may have 30 glutamines. But in the patients, they typically have 39 or more glutamines. So, imagine the difference between 30 and 39: If you just have a little bit more you're going to lose critical cells in the brain that coordinate balance.

So, we created animal models, we studied them, we identified the protein interactions of ataxin- 1 - the protein mutated in ataxia. I will summarize 20 years of work in the following discovery: We discovered that the glutamine expansion stabilizes the protein, makes it a little bit more stable, a little bit more abundant in the cell, leading to a little bit more enhanced interaction with its known normal native partners. You're basically having a little too much of something. We tested all that genetically and biochemically. We have a readout for ataxin-1 interactions. We can really show that those interactions are enhanced, and if you reduce the interactor by $50 \%$, you can now suppress the disease symptoms in animal models. It told us that we really needed to study what regulates ataxin-1.

(C) 2018 Zoghbi. This article is distributed under the terms of the Creative Commons Attribution-NonCommercial License, which permits reuse and redistribution, except for commercial purposes, provided that the original author and source are credited. 
We identified an RNA-binding protein that regulates it: The RNA-binding protein binds the untranslated portion of the RNA and suppresses the level of the RNA keeping the ataxin-1 protein in check. This is where we discovered you don't have to mutate ataxin-1 to cause toxicity.

We found that if we reduce the RNA-binding protein by $50 \%$, then ataxin- 1 levels go up by $20 \%$ or $30 \%$, and that increase of $20 \%$ or $25 \%$ of a wild-type ataxin- 1 induced Purkinje cell degeneration and balance problems. How are we sure it's that ataxin-1 25\% increase? Because we rely on genetics. We can now take these animals that lack one copy of the regulator, and therefore have a slightly higher ataxin-1, and breed them to mice that lack one copy of ataxin-1 to normalize ataxin-1, and you block the degeneration and all the symptoms for balance problems disappear. This pretty much for us nailed the importance of protein levels. Even a healthy protein can be toxic if increased by $20 \%$ or $30 \%$.

Jan Witkowski: I'm sure this offers therapeutic opportunities, but it also surely expands the number of ways in which one can get a neurodegenerative disease?

Dr. Zoghbi: Absolutely. We immediately recognized this offers a therapeutic opportunity, because if we can find the regulators of ataxin-1 that can be inhibited safely and for which you can design a small molecule, then we could perhaps develop a therapeutic. We set out to search for those using genetic screens in human cells and in collaboration with Juan Botas in fruit flies. We identified many candidate regulators that we are pursuing one by one, some of which we're now actually working with a pharmaceutical company to develop a small molecule therapeutic.

If you observe what is reported in the literature for other neurodegenerative diseases, you would find that what we have learned from this ataxia project applies to the more common neurodegenerative diseases? The genetic data tell us yes, because we know that doubling the levels of normal synuclein can cause Parkinson's. Tripling the level of normal synuclein can give you earlier disease onset. Doubling the amyloid precursor protein gene gives you dementia. People with Down syndrome will have an extra copy of amyloid precursor protein gene due to trisomy 21 and have onset of early Alzheimer's. We know for these genes and proteins, levels matter. You don't have to mutate any of them.

Jan Witkowski: In Down's...

Dr. Zoghbi: It's just extra APP [amyloid precursor protein] and we know it's the APP gene because there are a couple of individuals who have the trisomy but they lack the APP gene and they don't get Alzheimer's. Down individuals may get dementia at 35 or 40 , so it's really important for us to understand and figure out how to regulate APP levels.

Jan Witkowski: Why hasn't this been thought of before? Have people been so fixed on the idea that neurodegenerative disease, or any inherited disease, you have a mutation in a gene that produces a mutated protein and have ignored the fact that these fairly small differences in protein levels could have profound effects?
Dr. Zoghbi: I think people have thought about it, but have not pursued it necessarily as aggressively as needed. Clearly the duplications and the triplications, those were studied genetically and those were Mendelian-inherited disorders, although it's a wild-type protein. I think people are coming around now to realize there is another protein, tau, in which regulatory mutations or mutations that change the isoform ratio can also drive degeneration. This is where the lesson of ataxia got me closer to understanding at least one approach to consider for these common degenerative diseases. Let's find the regulators of these proteins, and let's think of ways we can slightly lower the levels of these diseasedriving proteins, and that might protect people. There are many ways we can get neurodegeneration, you're absolutely right. If you're a person with a duplication of APP, you're going to get early Alzheimer's. But you may be a person with healthy APP, a person that doesn't have any duplication but that may have a variant in a regulator of APP that's going to slightly increase APP. That might put someone at risk at 70. Finding the regulator is going to teach us more about the biology of these proteins, but also it's going to help us interpret DNA sequencing data.

Right now, we're hit with so many variants after genome sequencing. We have no idea which ones to study and why. We can put them in animal models and we might or might not get a phenotype. But if we have the framework that these 70 genes are known to regulate synuclein, these 100 regulate APP, and so on and so forth, then at least if you got a sequence variation in them, you have a functional assay that tells you this gene is important.

Jan Witkowski: Have people started looking into GWASs [genome-wide association studies] for regulatory genes of the sort that you're talking about?

Dr. Zoghbi: We just started, because we just really began to identify a large number of regulators that we're validating, and we are crossing these now with GWASs for Alzheimer's and Parkinson's. We're beginning to find overlap, but we still have to do more. We're just beginning to do that. For some, we're collaborating with Alison Goate and Rudy Tanzi; for others, we're using existing data that are publicly available.

Jan Witkowski: Do the sorts of regulators you're looking for have key signatures that enable you to say, "This sequence is for Regulator X?"

Dr. Zoghbi: I'll give you an example. We might have a kinase for a protein and if you phosphorylate that protein, you make that protein much more stable. For example, you prevent its degradation. That kinase becomes a candidate where a gain-of-function mutation may put someone at risk for neurodegeneration, whereas a loss-of-function heterozygosity might be neuroprotective.

Jan Witkowski: I think of all the kinases and all the phosphatases, and any one of these might be...

Dr. Zoghbi: A candidate, absolutely. The majority of people who don't have the family history don't have necessarily the risk to have a duplication of synuclein. But it's 
going to be something else, right? Because the pathology incriminates these proteins. These are at least some of the more common. If you took a hundred people with Alzheimer's, the vast majority of them will have abnormal APP accumulation or A $\beta$ [amyloid $\beta$ ] accumulation, abnormal tau phosphorylation, so you know they're effectors at some point in time, but there's no mutation in them. That's why I think the regulators are important.

Jan Witkowski: So, you can recognize effectors that are normal but producing clinical phenotypes. So then you say it's the level of the protein — not the mutation.

Dr. Zoghbi: Correct. It's what it's affecting. In the case of the ataxia, you remember the regulator I mentioned to you, that if we took one allele we saw elevation of ataxin-1 levels and we show in the mice, degeneration. We then found people with haploinsufficiency — with deletions or inactivating mutations of one allele - and these people have childhood ataxias and they have developmental disability and other neurological problems, but if they have even a milder mutation - not a total null allele in one allele-if they're haploinsufficient due to just a missense modulation, they have a late-onset balance disorder. So, you see the gradation of the phenotype.

Jan Witkowski: Certainly, there are therapeutic opportunities provided.

Dr. Zoghbi: If you actually knew that this is a big driver of disease, finding such regulators, studying them, and finding ways to elevate them or decrease them is going to be far more valuable.

Jan Witkowski: And that's only standard pharmacology to alter levels of things, as opposed to replacing a mutant gene.

Dr. Zoghbi: Exactly. So this is all with neurodegeneration. Now if we have a couple more minutes I'll tell you about childhood diseases. As you know, I've always been interested in Rett syndrome, and we discovered it is caused by mutations in the gene methyl-CpG-binding protein 2: $M E C P 2$. The function of MeCP2 protein is extremely critical for the brain and its levels really matter. If you have a mutation that totally inactivates the protein in a malebecause it's on the X chromosome - sadly, these males will be severely affected and they will die early. But if you have a mutation that's milder, that male will survive but will have neurological problems, and depending on the severity of the mutation their phenotype could be early autism, hyperactivity, or a little later if it's a milder muta- tion where he might present with schizophrenia, juvenileonset schizophrenia, or other behavioral problems. Here again you see gradation, from death in the first year of life with a null allele, all the way to milder psychiatric symptoms if the mutation is milder. If you have it in a girl, they're mosaic. It's on one of the two X chromosomes, so $50 \%$ of the cells are lacking a functional allele and this will cause Rett syndrome, which affects all aspects of brain function. On the other side of the coin, if you double the level of the protein, you get a progressive neurological syndrome. And if you triple it you get an even more severe phenotype. So here again, we're seeing that level/function phenotype relationship.

Jan Witkowski: The brain, presumably, is special in this case. Nerve cells are particularly sensitive.

Dr. Zoghbi: So far, that's what we're seeing. We're not noticing the effect of these proteins in dividing cells. We're seeing the sensitivity to protein dosage either in adult neurodegenerative diseases or in the childhood diseases like Rett syndrome. It seems that brain cells, once mature and they don't divide, they're probably much more vulnerable to changes in protein homeostasis. You need to get everything right.

Jan Witkowski: I seem to remember that the trinucleotide repeat disorders are preferentially brain-related.

Dr. Zoghbi: Right. At least the ones where the mutation is in the coding region are brain-specific. There may be some peripheral phenotype in the spinal bulbar muscular atrophy, but that's really not what drives the disease. It's the brain-specific — or nerve cell-specific, because here it's the spinal cord. There's some that affect muscles and other tissues, but these are outside the coding region, so the expansion is not within the protein.

Jan Witkowski: Fascinating. And a bit depressing, thinking of all the extra things that could go wrong.

Dr. Zoghbi: I actually find it more optimistic if we know now that protein levels matter. That means we have an opportunity to find things that regulate these disease-driving proteins, and any such opportunity could be translated into therapy, whether small-molecule, or antisense oligonucleotides. I think new DNA-encoded libraries can be used to target almost any protein. You don't have to have an enzyme to have a therapeutic anymore. The new libraries that one can use screening billions of compounds could really reveal compounds that might regulate any protein product. 


\section{$\$_{\text {CSH\& }}^{\infty}$ Cold Spring Harbor Symposia SYMPOSIA}

\section{A Conversation with Huda Zoghbi}

Cold Spring Harb Symp Quant Biol 2018 83: 293-295 originally published online April 23, 2019 Access the most recent version at doi:10.1101/sqb.2018.83.037721

Creative This article is distributed under the terms of the

Commons http://creativecommons.org/licenses/by-nc/4.0/, which permits reuse and

License redistribution, except for commercial purposes, provided that the original author and source are credited.

Email Alerting Receive free email alerts when new articles cite this article - sign up in Service the box at the top right corner of the article or click here. 